\title{
Endpoint estimates for the commutators of strongly singular integral operators
}

\author{
Huihui Zhang ${ }^{\mathrm{a}}$, Xiao $\mathrm{Yu}^{\mathrm{a}, *}$, Xiaomei $\mathrm{Wu}^{\mathrm{b}}$ \\ a College of Mathematics and Computer Science, Shangrao Normal University, 401 Zhimin St., \\ Xinzhou Dist., Shangrao 334001, Jiangxi Prov., P.R. China \\ b College of Xingzhi, Zhejiang Normal University, 688 Yingbin St., Wucheng Dist., Jinhua 321004, \\ ZheJiang Prov., P.R. China
}

*Corresponding author, e-mail: yx2000s@163.com

Received 26 Aug 2017

Accepted 24 Aug 2018

\begin{abstract}
In this paper, we give sufficient and necessary conditions for the endpoint estimates of the commutators
\end{abstract} generated by the strongly singular integrals and the BMO function on the extreme case.

KEYWORDS: strongly singular integral, commutator, BMO space, $H^{1}$ space, endpoint estimates

MSC2010: 42B20, 42B30

\section{INTRODUCTION}

It is well-known that the theory of the strongly Calderón-Zygmund operator was originated from a class of multiplier operators with its symbol given by $e^{i|\xi|^{a}} /|\xi|^{\beta}$ with $0<a<1$ and $\beta>0$. In 1972 , Fefferman and Stein ${ }^{1}$ enlarged the multiplier operators onto a class of convolution operators. Later, Coifman $^{2}$ studied a related class of operators in the case $n=1$.

In 1986, Alvarez and Milman ${ }^{3}$ introduced a class of non-convolution operator whose kernel is more singular near the diagonal than those of the classical Calderón-Zygmund operators.

Definition 1 (Ref. 3) Let $T: \mathscr{S} \rightarrow \mathscr{S}^{\prime}$ be a bounded linear operator. $T$ is called a strongly singular Calderón-Zygmund operator if the following conditions are fulfilled:

(i) $T$ extends to a continuous operator from $L^{2}$ into itself.

(ii) $T$ is associated with a certain standard kernel. More precisely, there exists a function $K(x, y)$ continuous on the diagonal of $\mathbb{R}^{2 n}$ such that $|K(x, y)-K(x, z)|+|K(y, x)-K(z, x)| \leqslant$ $C|y-z|^{\delta} /|x-z|^{n+\delta / \alpha}$ with $2|y-z|^{\alpha} \leqslant|x-z|$ for some $0<\delta \leqslant 1,0<\alpha<1$ and $\langle T f, g\rangle=$ $\int K(x, y) f(y) g(x) d x d y$ for $f, g \in \mathscr{S}$ with disjoint supports.

(iii) For some $\frac{(1-\alpha) n}{2} \leqslant \beta<\frac{n}{2}$, both operators $T$ and $T^{*}$ extend to continuous operators from $L^{q}$ to $L^{2}$ with $\frac{1}{q}=\frac{1}{2}+\frac{\beta}{n}$ and $1<q<2$. (iv) From (iii), we know that $T$ also extends to a continuous operator from $L^{2}$ to $L^{q^{\prime}}$ with $\frac{1}{q^{\prime}}=$ $1-\frac{1}{q}=\frac{1}{2}-\frac{\beta}{n}$.

Alvarez and Milman ${ }^{3}$ proved that the strongly singular Calderón-Zygmund operator $T$ is bounded from $L^{\infty}$ to BMO and from $L^{1}$ to $L^{1, \infty}$. Here, $\mathrm{BMO}$ is the bounded mean oscillation space and its definition can be stated as follows.

Definition 2 (Ref. 4) A function $f$ is said to belong to $\operatorname{BMO}\left(\mathbb{R}^{n}\right)$ if the following sharp maximal function is bounded

$$
f^{\sharp}(x)=\sup _{B \ni x} \frac{1}{|B|} \int_{B}\left|f(y)-f_{B}\right| d y<\infty,
$$

where the supreme is taken over all balls $B \subset \mathbb{R}^{n}$. Moreover, $f_{B}=\frac{1}{|B|} \int_{B} f(x) d x$ and $\|f\|_{\mathrm{BMO}}=\left\|f^{\sharp}\right\|_{L^{\infty}}$.

Then, using the interpolation theory, we know that $T$ is bounded on $L^{p}$ space with $1<p<\infty$. Moreover, Alvarez and Milman ${ }^{3}$ gave the estimates of the sharp maximal function $(T f)^{\sharp}$, which implies that the weighted norm estimates for $T$ can be obtained. Thus, by the well-known theorem proved by Alvarez, Bagby, Kurtz and Pérez in Ref. 5, we have the following theorem.

Theorem 1 (Ref. 6) Suppose that $T_{b}$ is the commutator generated by the strongly singular CalderónZygmund operator $T$ and a BMO function $b$. Then, 
$T_{b}$ is bounded on $L^{p}\left(\mathbb{R}^{n}\right)$ with $1<p<\infty$ and the definition of $T_{b}$ is defined by

$$
T_{b} f(x)=b(x) T(f)(x)-T(b f)(x) .
$$

Here we would like to mention that the operator $T_{b}$ was originated from the commutators of the classical C-Z singular integral operators which was proposed by Coifman, Rochberg and Weiss ${ }^{7}$ in 1976.

In 1995, Pérez ${ }^{8}$ gave a counterexample to show that the commutator of the C-Z singular integral is not bounded from $H^{1}$ to $L^{1}$. Later, Harboure, Segovia and Torrea ${ }^{9}$ gave sufficient and necessary conditions for the endpoint estimates of the commutator generated by the C-Z singular integrals on $L^{\infty}$ and $H^{1}$ spaces.

On the other hand, for the study of commutators generated by the strongly singular integral operators and BMO functions, one may see Refs. 10-12 for more details. However, the sufficient and necessary conditions for the endpoint estimates of $T_{b}$ is still unknown. In this paper, we will give a positive answer to this question.

Before giving the main results of this paper, we give the atomic decomposition of $H^{1}$ space. For more details about $H^{1}$ space, one may see Ref. 1 .

Definition 3 (Ref. 1) We say a function $a(x)$ is an atom of $H^{1}$ if $a$ satisfies the following conditions

(i) $\operatorname{supp}(a) \subset B\left(x_{0}, r\right)$,

(ii) $\|a\| L^{\infty} \leqslant\left|B\left(x_{0}, r\right)\right|^{-1}$,

(iii) $\int a(x) d x=0$.

It is well-known that if a function $f$ belongs to $H^{1}$, then it can be written as $f=\sum_{i=1}^{\infty} \lambda_{i} a_{i}$ where each $a_{i}$ is a $H^{1}$ atom. Moreover, we have

$$
\|f\|_{H^{1}} \sim \inf \left\{\sum_{i=-\infty}^{+\infty}\left|a_{i}\right|\right\}
$$

where the infimum is taken over all decompositions of $f$.

Our results can be stated as follows.

Theorem 2 Let $\alpha, \beta$ and $\delta$ be the same as in Definition 1. Suppose that $T_{b}$ is a commutator generated by the strongly singular integral operator and a BMO function $b$, then the following two conditions are equivalent:

(i) $T_{b}$ is bounded from $L^{\infty}$ to $B M O$. (ii) For any cube $Q=Q(z, r)$, if $r>1$,

$$
\begin{aligned}
& \left(\frac{1}{|Q|} \int_{Q}\left|b(x)-b_{Q}\right| d x\right) \\
& \times\left|\int_{(Q(z, 2 \sqrt{n} r))^{c}} K(z, y) f(y) d y\right| \leqslant C\|f\|_{L^{\infty}},
\end{aligned}
$$

where the constant $C$ only depends on $n, \alpha, \beta$ and $\delta$. On the other hand, for the case $0<\epsilon \leqslant r \leqslant 1$ with any $\epsilon>0$,

$$
\begin{aligned}
& \left(\frac{1}{|Q|} \int_{Q}\left|b(x)-b_{Q\left(z, 2 \sqrt{n} r^{\alpha}\right)}\right| d x\right) \\
& \times\left|\int_{Q\left(z, 2 \sqrt{n} r^{\alpha}\right)^{c}} K(z, y) f(y) d y\right| \leqslant C\|f\|_{L^{\infty}},
\end{aligned}
$$

where the constant $C$ only depends on $n, \alpha, \beta, \delta$ and $\epsilon$.

Theorem 3 Let $\alpha, \beta$ and $\delta$ be the same as in Definition 1. Suppose that $T_{b}$ is a commutator generated by the strongly singular integral operator and a BMO function $b$, then the following two conditions are equivalent:

(i) $T_{b}$ is bounded from $H^{1}$ to $L^{1}$.

(ii) For any $H^{1}$ atom $a(x)$ supported on $Q(z, r)$, if $r>1$,

$$
\left|\int_{Q(z, 2 \sqrt{n} r)^{c}} K(x, z) d x \int_{Q} b(y) a(y) d y\right| \leqslant C,
$$

where the constant $C$ only depends on $n, \alpha, \beta$ and $\delta$. On the other hand, for the case $0<\epsilon \leqslant r \leqslant 1$ with any $\epsilon>0$, there is

$$
\left|\int_{Q\left(z, 2 \sqrt{n} r^{\alpha}\right)^{c}} K(x, z) d x \int_{Q} b(y) a(y) d y\right| \leqslant C,
$$

where the constant $C$ only depends on $n, \alpha, \beta, \delta$ and $\epsilon$.

\section{ENDPOINT ESTIMATES FOR $T_{b}$ FROM $L^{\infty}$ TO BMO SPACE.}

Before giving the proof of Theorem 2, we give some lemmas that will be very useful throughout this paper.

Lemma 1 (Ref. 4) Let $1<p<\infty$, and let $f \in$ $\operatorname{BMO}\left(\mathbb{R}^{n}\right)$, then we have

(i) $\|f\|_{\mathrm{BMO}} \sim \sup _{B}\left(\frac{1}{|B|} \int_{B}\left|f(x)-f_{B}\right|^{p} d x\right)^{1 / p}$.

(ii) $\|f\|_{\mathrm{BMO}} \sim \sup _{B} \inf _{a \in R} \frac{1}{|B|} \int_{B}|f(x)-a| d x$. 
Lemma 2 (Ref. 4) Let $f \in \mathrm{BMO}\left(\mathbb{R}^{n}\right)$, and let $1 \leqslant q<$ have $\infty$ and $r_{1}, r_{2} \in \mathbb{R}^{+}$, then

$$
\begin{aligned}
& \left(\frac{1}{\left|B\left(z, r_{1}\right)\right|} \int_{B\left(z, r_{1}\right)}\left|f(x)-f_{B\left(z, r_{2}\right)}\right|{ }^{q} d x\right)^{1 / q} \\
& \quad \leqslant C\left(1+\left|\log \frac{r_{1}}{r_{2}}\right|\right)\|f\|_{\mathrm{BMO}}
\end{aligned}
$$

for any $z \in \mathbb{R}^{n}$.

Now, we are going to give the proof of Theorem 2 and we may consider this problem into the case $r>1$ and $0<\epsilon \leqslant r \leqslant 1$.

\section{(i) The case $r>1$}

For any cube $Q=Q(z, r)$ with $r>1$, splitting $f$ as $f=f_{1}+f_{2}$ with $f_{1}(x)=f(x) \chi_{Q(z, 2 \sqrt{n} r)}$. Then using some basic ideas from Ref. 9 (p. 680), we denote

$$
\begin{aligned}
\sigma_{1}(x) & =T_{b} f_{1}(x), \\
\sigma_{2}(x, z) & =\left(b(x)-b_{Q}\right)\left(T f_{2}(x)-T f_{2}(z)\right), \\
\sigma_{3}(x, z) & =T\left(\left(b-b_{Q}\right) f_{2}\right)(z)-T\left(\left(b-b_{Q}\right) f_{2}\right)(x), \\
\sigma_{4}(x, z) & =\left(b(x)-b_{Q}\right) T f_{2}(z) .
\end{aligned}
$$

$$
\begin{aligned}
& \left|T f_{2}(x)-T f_{2}(z)\right| \\
& \quad \leqslant \int_{|y-z| \geqslant 2 \sqrt{n} r}|K(x, y)-K(z, y)| \times|f(y)| d y \\
& \quad \leqslant C\|f\|_{L^{\infty}} \int_{|y-z| \geqslant 2 \sqrt{n} r} \frac{|x-z|^{\delta}}{|y-z|^{n+\frac{\delta}{\alpha}}} d y \\
& \quad \leqslant C\|f\|_{L^{\infty}} r^{\delta} \int_{2 \sqrt{n} r}^{\infty} r^{n-1} r^{-n-\frac{\delta}{\alpha}} d r \\
& \quad \leqslant C\|f\|_{L^{\infty}} r^{\delta\left(1-\frac{1}{\alpha}\right)} \leqslant C\|f\|_{L^{\infty}},
\end{aligned}
$$

where the last inequality follows from $r^{\delta\left(1-\frac{1}{\alpha}\right)} \leqslant$ $r^{0}=1$. Thus, we obtain

Thus, we may split $T_{b} f(x)-\left(T_{b} f\right)_{Q}$ as

$$
\begin{aligned}
T_{b} f(x)-\left(T_{b} f\right)_{Q}= & \sigma_{1}(x)-\left(\sigma_{1}\right)_{Q}+\sigma_{2}(x, z) \\
& +\sigma_{4}(x, z)-\left(\sigma_{2}(\cdot, z)\right)_{Q} \\
& +\left(\sigma_{3}(x, \cdot)\right)_{Q} .
\end{aligned}
$$

Next, we will give the estimates of $\frac{1}{|Q(z, r)|} \int_{Q(z, r)}\left|\sigma_{i}\right| d x \quad(i=1,2,3), \quad$ respectively. For $\sigma_{1}(x)$, from (i) of Definition 1 and Theorem 1, we have

$$
\begin{aligned}
& \frac{1}{|Q(z, r)|} \int_{Q(z, r)}\left|\sigma_{1}(x)\right| d x \\
& \quad=\frac{1}{|Q(z, r)|} \int_{Q(z, r)}\left|T_{b} f_{1}(x)\right| d x \\
& \quad \leqslant \frac{1}{|Q(z, r)|}\left\|T_{b} f_{1}\right\|_{2}|Q(z, r)|^{\frac{1}{2}} \\
& \quad \leqslant C\left\|f_{1}\right\|_{L^{2}}|Q(z, r)|^{-1 / 2} \\
& \quad \leqslant C\|f\|_{L^{\infty}}|Q(z, 2 \sqrt{n} r)|^{1 / 2}|Q(z, r)|^{-1 / 2} \\
& \quad \leqslant C\|f\|_{L^{\infty}} .
\end{aligned}
$$

To estimate $\sigma_{2}$, we give the following estimates. For any $x \in Q(z, r)$ and $y \in Q(z, 2 \sqrt{n} r)^{c}$, there is $2|x-z|^{\alpha} \leqslant 2(\sqrt{n} r)^{\alpha} \leqslant 2 \sqrt{n} r \leqslant|y-z|$ with $r>1$ and $0<\alpha<1$. Then, using (ii) of Definition 1, we

As $0<\alpha<1$, we have $2|x-z|^{\alpha} \leqslant 2(\sqrt{n} r)^{\alpha} \leqslant$ $2 \sqrt{n} r \leqslant|y-z|$. Then, using (ii) of Definition 1 and 
Lemma 2, we get

$$
\begin{aligned}
& \left|\sigma_{3}(x, z)\right| \leqslant C\|f\|_{L^{\infty}} \int_{|y-z| \geqslant 2 \sqrt{n} r}\left|b(y)-b_{Q}\right| \\
& \times \frac{|x-z|^{\delta}}{|y-z|^{n+\delta / \alpha}} d y \\
& \leqslant C\|f\|_{L^{\infty}} \sum_{j=0}^{\infty} \int_{2^{j \cdot 2 \sqrt{n} r} \leqslant|y-z|<2^{j+1} \cdot 2 \sqrt{n} r} \\
& \left|b(y)-b_{Q}\right| \frac{|x-z|^{\delta}}{|y-z|^{n+\delta / \alpha}} d y \\
& \leqslant C\|f\|_{L^{\infty}} \sum_{j=0}^{\infty} \frac{r^{\delta}}{\left(2^{j} \cdot 2 \sqrt{n} r\right)^{n+\delta / \alpha}} \\
& \times \int_{2^{j \cdot 2 \sqrt{n} r} \leqslant|y-z|<2^{j+1} \cdot 2 \sqrt{n} r}\left|b(y)-b_{Q}\right| d y \\
& \leqslant C\|b\|_{\mathrm{BMO}}\|f\|_{L^{\infty}} \sum_{j=0}^{\infty} \frac{r^{\delta} \cdot\left(2^{j+1} 2 \sqrt{n} r\right)^{n}}{\left(2^{j} \cdot 2 \sqrt{n} r\right)^{n+\delta / \alpha}}(j+1) \\
& \leqslant C\|b\|_{\text {BMO }}\|f\|_{L^{\infty}} r^{\delta\left(1-\frac{1}{\alpha}\right)} \sum_{j=0}^{\infty}\left(2^{j}\right)^{-\delta / \alpha}(j+1) \\
& \leqslant C\|b\|_{\mathrm{BMO}}\|f\|_{L^{\infty}} r^{0}=C\|b\|_{\mathrm{BMO}}\|f\|_{L^{\infty}},
\end{aligned}
$$

mates of $\frac{1}{|Q|} \int_{Q}\left|\sigma_{1}(x)\right| d x$ as$$
:=I+I I \text {. }
$$
For $I$, by using the Hölder inequality, the fact that $0<\epsilon \leqslant r \leqslant 1$, the $L^{2} \rightarrow L^{q^{\prime}}$ boundedness of $T$ ((iv) of Definition 1) and Lemma 2, we get

which implies

$$
\frac{1}{|Q(z, r)|} \int_{Q(z, r)}\left|\sigma_{3}(x, z)\right| d x \leqslant C\|b\|_{\mathrm{BMO}}\|f\|_{L^{\infty}} .
$$

\section{(ii) The case $0<\epsilon \leqslant r \leqslant 1$}

For the case $0<\epsilon \leqslant r \leqslant 1$ with any $\epsilon>0$, splitting $f$ as $f=f_{1}+f_{2}$ with $f_{1}=f \chi_{Q\left(z, 2 \sqrt{n} r^{\alpha}\right)}$. Then, following some basic ideas from the case $r>1$, we may denote $\sigma_{1}, \sigma_{2}, \sigma_{3}$ and $\sigma_{4}$ as follows.

$$
\begin{aligned}
\sigma_{1}(x)= & T_{b} f_{1}(x) \\
\sigma_{2}(x, z)= & \left(b(x)-b_{Q\left(z, 2 \sqrt{n} r^{\alpha}\right)}\right)\left(T f_{2}(x)-T f_{2}(z)\right), \\
\sigma_{3}(x, z)= & T\left(\left(b-b_{Q\left(z, 2 \sqrt{n} r^{\alpha}\right)}\right) f_{2}\right)(z) \\
& -T\left(\left(b-b_{Q}\right) f_{2}\right)(x) \\
\sigma_{4}(x, z)= & \left(b(x)-b_{Q\left(z, 2 \sqrt{n} r^{\alpha}\right)}\right) T f_{2}(z) .
\end{aligned}
$$

Thus, we have

$$
\begin{aligned}
T_{b} f(x)-\left(T_{b} f\right)_{Q\left(z, 2 \sqrt{n} r^{\alpha}\right)} & \\
= & \sigma_{1}(x)-\left(\sigma_{1}\right)_{Q\left(z, 2 \sqrt{n} r^{\alpha}\right)}+\sigma_{2}(x, z)+\sigma_{4}(x, z) \\
& -\left(\sigma_{2}(\cdot, z)\right)_{Q\left(z, 2 \sqrt{n} r^{\alpha}\right)}+\left(\sigma_{3}(x, \cdot)\right)_{Q\left(z, 2 \sqrt{n} r^{\alpha}\right)} .
\end{aligned}
$$

Next, we will estimate $\frac{1}{|Q|} \int_{Q}\left|\sigma_{i}\right| d x$ with $i=$ $1,2,3$, respectively. For $\sigma_{1}$, we may give the esti-

$$
\begin{aligned}
\frac{1}{|Q|} \int_{Q}\left|\sigma_{1}(x)\right| d x \\
=\frac{1}{|Q|} \int_{Q}\left|T\left((b(x)-b(\cdot)) f_{1}\right)(x)\right| d x \\
=\frac{1}{|Q|} \int_{Q}\left|T\left(f_{1}\right)(x)\right|\left|b(x)-b_{Q\left(z, 2 \sqrt{n} r^{\alpha}\right)}\right| d x \\
\quad+\frac{1}{|Q|} \int_{Q}\left|T\left(\left(b_{Q\left(z, 2 \sqrt{n} r^{\alpha}\right)}-b(\cdot)\right) f_{1}\right)(x)\right| d x
\end{aligned}
$$

$$
\begin{aligned}
I= & \frac{1}{|Q|} \int_{Q}\left|T\left(f_{1}\right)(x)\right|\left|b(x)-b_{Q\left(z, 2 \sqrt{n} r^{\alpha}\right)}\right| d x \\
\leqslant & \frac{|Q|^{1 / q}}{|Q|} \times\left(\frac{1}{|Q|} \int_{Q}\left|b(x)-b_{Q\left(z, 2 \sqrt{n} r^{\alpha}\right)}\right|^{q} d x\right)^{1 / q} \\
& \times\left(\int_{Q}\left|T f_{1}(x)\right|^{q^{\prime}} d x\right)^{1 / q^{\prime}} \\
\leqslant & C\|b\|_{\mathrm{BMO}}\left\|f_{1}\right\|_{L^{2}} \frac{|Q|^{1 / q}}{|Q|} \\
\leqslant & C\|b\|_{\mathrm{BMO}}\|f\|_{L^{\infty}}\left|Q\left(z, 2 \sqrt{n} r^{\alpha}\right)\right|^{1 / 2} \frac{|Q|^{1 / q}}{|Q|} \\
\leqslant & C\|b\|_{\mathrm{BMO}}\|f\|_{L^{\infty}} r^{n\left(\frac{\alpha}{2}+\frac{1}{q}-1\right)} \\
\leqslant & C\|b\|_{\mathrm{BMO}}\|f\|_{L^{\infty}}
\end{aligned}
$$

where the last inequality follows from the fact $0<$ $r \leqslant 1$ and $\frac{\alpha}{2}+\frac{1}{q}-1=\frac{\alpha}{2}-1+\frac{1}{2}+\frac{\beta}{n} \geqslant \frac{\alpha}{2}-\frac{1}{2}+\frac{1}{2}-\frac{\alpha}{2}=0$. For $I I$, using Lemma 2, the Hölder inequality 
and the $L^{2} \rightarrow L^{q^{\prime}}$ boundedness of $T$, we have

$$
\begin{aligned}
I I= & \frac{1}{|Q|} \int_{Q}\left|T\left(\left(b_{Q\left(z, 2 \sqrt{n} r^{\alpha}\right)}-b(\cdot)\right) f_{1}\right)(x)\right| d x \\
\leqslant & \frac{1}{|Q|}\left\|T\left(\left(b_{Q\left(z, 2 \sqrt{n} r^{\alpha}\right)}-b(\cdot) f_{1}(\cdot)\right)\right)\right\|_{L^{q^{\prime}}}|Q|^{1 / q} \\
\leqslant & \frac{1}{|Q|}\left\|\left(b_{Q\left(z, 2 \sqrt{n} r^{\alpha}\right)}-b(\cdot)\right) f_{1}(\cdot)\right\|_{L^{2}}|Q|^{1 / q} \\
\leqslant & \|f\|_{L^{\infty}}|Q|^{-1 / q^{\prime}} \\
& \times\left(\int_{Q\left(z, 2 \sqrt{n} r^{\alpha}\right)}\left|b_{Q\left(z, 2 \sqrt{n} r^{\alpha}\right)}-b(x)\right|^{2} d x\right)^{1 / 2} \\
\leqslant & C\|f\|_{L^{\infty}|Q|^{-1 / q^{\prime}}\left|Q\left(z, 2 \sqrt{n} r^{\alpha}\right)\right|^{1 / 2}} \\
& \times\left(\frac{1}{\left|Q\left(z, 2 \sqrt{n} r^{\alpha}\right)\right|} \int_{Q\left(z, 2 \sqrt{n} r^{\alpha}\right)}\right. \\
\leqslant & C\|b\|_{\mathrm{BMO}}\|f\|_{L^{\infty}} r^{n(\alpha / 2+1 / q-1)} \\
= & C\|b\|_{\mathrm{BMO}}\|f\|_{L^{\infty}} .
\end{aligned}
$$

Combing the above two estimates, we get

$$
\frac{1}{|Q|} \int_{Q}\left|\sigma_{1}(x)\right| d x \leqslant C\|b\|_{\text {ВMо }}\|f\|_{L^{\infty}} .
$$

Next, we give the estimate of $\sigma_{2}(x, z)$. As $y \in$ $Q\left(z, 2 \sqrt{n} r^{\alpha}\right)$ and $x \in Q(z, r)$, there is $2|x-z|^{\alpha} \leqslant$ $2 \sqrt{n} r^{\alpha} \leqslant|y-z|$. Thus, we obtain

$$
\begin{aligned}
& \left|T f_{2}(x)-T f_{2}(z)\right| \\
& \quad \leqslant \int_{\mathbb{R}^{n}}|K(x, y)-K(z, y)| f_{2}(y) \mid d y \\
& \quad \leqslant \int_{|y-z| \geqslant 2 \sqrt{n} r r^{\alpha}}\left|K(x, y)-K(z, y) \| f_{2}(y)\right| d y \\
& \quad \leqslant\|f\|_{L^{\infty}} \int_{|y-z| \geqslant 2 \sqrt{n} r^{\alpha}} \frac{|x-z|^{\delta}}{|y-z|^{n+\frac{\delta}{\alpha}}} d y \\
& \quad \leqslant C\|f\|_{L^{\infty}} r^{\delta} \int_{2 \sqrt{n} r^{\alpha}}^{\infty} t^{n-1} t^{-n-\frac{\delta}{\alpha}} d t \leqslant C\|f\|_{L^{\infty}} .
\end{aligned}
$$

Using Lemma 2 and the fact $0<\epsilon \leqslant \alpha<1$, we get

$$
\begin{aligned}
& \frac{1}{|Q|} \int_{Q}\left|\sigma_{2}(x, z)\right| d x \\
& \quad \leqslant C\|f\|_{L^{\infty}} \frac{1}{|Q|} \times \int_{Q}\left|b(x)-b_{Q\left(z, 2 \sqrt{n} r^{\alpha}\right)}\right| d x \\
& \quad \leqslant C\|b\|_{\text {BMO }}\|f\|_{L^{\infty}}
\end{aligned}
$$

where the last inequalities follows from the fact $0<$ $\epsilon \leqslant r \leqslant 1$.
For $\sigma_{3}(x, z)$, by the fact $2|x-z|^{\alpha} \leqslant 2 \sqrt{n} r^{\alpha} \leqslant$ $|y-z|$, Lemma 2 and (ii) of Definition 1, we get

$$
\begin{aligned}
& \left|\sigma_{3}(x, z)\right|=\mid \int_{\mathbb{R}^{n}}\left(b(y)-b_{Q\left(z, 2 \sqrt{n} r^{\alpha}\right)}\right) \\
& \times f_{2}(y)(K(z, y)-K(x, y)) d y \mid \\
& \leqslant \int_{|y-z| \geqslant 2 \sqrt{n} r^{\alpha}}\left|b(y)-b_{Q\left(z, 2 \sqrt{n} r^{\alpha}\right)}\right| \\
& \times|f(y)||K(x, y)-K(z, y)| d y \\
& \leqslant C\|f\|_{L^{\infty}} \int_{|y-z| \geqslant 2 \sqrt{n} r^{\alpha}}\left|b(y)-b_{Q\left(z, 2 \sqrt{n} r^{\alpha}\right)}\right| \\
& \times \frac{|x-z|^{\delta}}{|y-z|^{n+\frac{\delta}{\alpha}}} d y \\
& \leqslant C\|f\|_{L^{\infty}} \sum_{j=0}^{\infty} \int_{2^{j \cdot 2 \sqrt{n}} r^{\alpha} \leqslant|y-z|<2^{j+1} \cdot 2 \sqrt{n} r^{\alpha}} \\
& \left|b(y)-b_{Q\left(z, 2 \sqrt{n} r^{\alpha}\right)}\right| \frac{|x-z|^{\delta}}{|y-z|^{n+\frac{\delta}{\alpha}}} d y \\
& \leqslant C\|f\|_{L^{\infty}} \sum_{j=0}^{\infty} \frac{r^{\delta}}{\left(2^{j} \cdot 2 \sqrt{n} r^{\alpha}\right)^{n+\frac{\delta}{\alpha}}} \\
& \times \int_{2^{j} \cdot 2 \sqrt{n} r^{a} \leqslant|y-z|<2^{j+1} \cdot 2 \sqrt{n} r^{a}} \\
& \left|b(y)-b_{Q\left(z, 2 \sqrt{n} r^{\alpha}\right)}\right| d y \\
& \leqslant C\|b\|_{\mathrm{BMO}}\|f\|_{L^{\infty}} \\
& \times \sum_{j=0}^{\infty} \frac{r^{\delta}\left(2^{j+1} \cdot 2 \sqrt{n} r^{\alpha}\right)^{n}(j+1)}{\left(2^{j+1} \cdot 2 \sqrt{n} r^{\alpha}\right)^{n+\frac{\delta}{\alpha}}} \\
& \leqslant C\|b\|_{\mathrm{BMO}}\|f\|_{L^{\infty}} \sum_{j=0}^{\infty} 2^{-\frac{\delta}{\alpha}(j+1)}(j+1) \\
& \leqslant C\|b\|_{\mathrm{BMO}}\|f\|_{L^{\infty}} \text {, }
\end{aligned}
$$

which implies

$$
\frac{1}{|Q|} \int_{Q}\left|\sigma_{3}(x, z)\right| d x \leqslant C\|b\|_{\mathrm{BMO}}\|f\|_{L^{\infty}} .
$$

\section{Proof of Theorem 2}

Now we are ready to give the proof of Theorem 2 . For any cube $Q=Q(z, r)$ with $r>1$, as $\sigma_{4}(x, z)=$ $T_{b} f(x)-\left(T_{b}(f)\right)_{Q}-\sigma_{1}(x)+\left(\sigma_{1}\right)_{Q}-\sigma_{2}(x, z)+$ $\left(\sigma_{2}(\cdot, z)\right)_{Q}-\left(\sigma_{3}(x, \cdot)\right)_{Q}$. From (1)-(3), we find that if $T_{b}$ is bounded from $L^{\infty}$ to BMO, then it is easy to get

$$
\frac{1}{|Q|} \int_{Q}\left|\sigma_{4}(x, z)\right| d x \leqslant C\|b\|_{\text {BMO }}\|f\|_{L^{\infty}}
$$


By the definition of $\sigma_{4}$, (7) is equivalent to

$$
\begin{aligned}
& \frac{1}{|Q|} \int_{Q}\left|b(x)-b_{Q}\right| d x \\
& \quad \times\left|\int_{(Q(z, 2 \sqrt{n} r))^{c}} K(z, y) f(y) d y\right| \leqslant C\|f\|_{L^{\infty}} .
\end{aligned}
$$

On the other hand, if (8) holds, it is clear that $T_{b}$ is bounded from $L^{\infty}$ to BMO.

Similarly, for any cube $Q=Q(z, r)$ with $0<\epsilon \leqslant$ $r \leqslant 1$, using -, it is easy to see that the boundedness of $T_{b}$ from $L^{\infty}$ to $\mathrm{BMO}$ is equivalent to

$$
\begin{aligned}
& \left(\frac{1}{|Q|} \int_{Q}\left|b(x)-b_{Q\left(z, 2 \sqrt{n} r^{\alpha}\right)}\right| d x\right) \\
& \quad \times\left|\int_{\left(Q\left(z, 2 \sqrt{n} r^{\alpha}\right)\right)^{c}} K(z, y) f(y) d y\right| \leqslant C\|b\|_{\mathrm{BMO}}\|f\|_{L^{\infty}} .
\end{aligned}
$$

Thus, the proof of Theorem 2 has been finished.

\section{ENDPOINT ESTIMATES FOR $T_{b}$ FROM $H^{1}$ TO $L^{1}$ SPACES.}

In this section, we will give the proof of Theorem 3 . For any $H^{1}$ atom $a(x)$ supported on $Q=Q(z, r)$, we will estimate $T_{b} a(x)$ in two cases.

\section{(i) The case $r>1$}

For the case $r>1$, we may decompose $T_{b} a(x)$ by

$$
\begin{aligned}
T_{b} a(x)= & T_{b} a(x) \chi_{Q(z, 2 \sqrt{n} r)}(x) \\
& +T_{b} a(x) \chi_{(Q(z, 2 \sqrt{n} r)) c}(x) \\
= & T_{b} a(x) \chi_{Q(z, 2 \sqrt{n} r)}(x) \\
& +T\left(a(\cdot)\left(b(\cdot)-b_{Q}\right)\right)(x) \chi_{(Q(z, 2 \sqrt{n} r)) c}(x) \\
& +\chi_{(Q(z, 2 \sqrt{n} r)) c}(x) T\left(\left(b(\cdot)-b_{Q}\right) a\right)(x) \\
= & T_{b} a(x) \chi_{Q(z, 2 \sqrt{n} r)}(x) \\
& +T\left(a(\cdot)\left(b(\cdot)-b_{Q}\right)\right)(x) \chi_{(Q(z, 2 \sqrt{n} r)) c}(x) \\
& +\chi_{(Q(z, 2 \sqrt{n} r)) c}(x) \int_{Q}(K(x, y)-K(x, z)) \\
& \times\left(b(y)-b_{Q}\right) a(y) d y+\chi_{(Q(z, 2 \sqrt{n} r)) c}(x) \\
& \times \int_{Q} K(x, z)\left(b(y)-b_{Q}\right) a(y) d y \\
:= & \mu_{1}(x)+\mu_{2}(x)+\mu_{3}(x, z)+\mu_{4}(x, z) .
\end{aligned}
$$

Then we will give the estimates of $\left\|\mu_{i}(\cdot)\right\|_{L^{1}}(i=$ $1,2,3)$, respectively. For $\mu_{1}$, by Theorem 1 and the
Hölder inequality, we have

$$
\begin{aligned}
\left\|\mu_{1}(\cdot)\right\|_{L^{1}} & =\left\|T_{b}(a)\right\|_{L^{1}(Q(z, 2 \sqrt{n} r))} \\
& =\int_{Q(z, 2 \sqrt{n} r)}\left|T_{b}(a)(x)\right| d x \\
& \leqslant C|Q(z, 2 \sqrt{n} r)|^{1 / 2}\left(\int_{\mathbb{R}^{n}}\left|T_{b}(a)(x)\right|^{2} d x\right)^{1 / 2} \\
& \leqslant C r^{\frac{n}{2}}\|a\|_{L^{2}} \\
& \leqslant C|Q|^{1 / 2}|Q|^{-1 / 2} \leqslant C .
\end{aligned}
$$

For $\mu_{2}$, as $x \in Q(z, 2 \sqrt{n} r)^{c}$ and $y \in Q(z, r)$, there is $2|y-z|^{\alpha} \leqslant 2 \sqrt{n} r^{\alpha} \leqslant 2 \sqrt{n} r \leqslant|x-z|$. Moreover, by the cancellation condition of $a$ and (ii) of Definition 1, we obtain

$$
\begin{aligned}
\left\|\mu_{2}(\cdot)\right\|_{L^{1}} & \left(b(x)-b_{Q}\right) \int_{Q} K(x, y) a(y) d y d x \\
= & \int_{Q(z, 2 \sqrt{n} r)^{c}}\left(b(x)-b_{Q}\right) \\
= & \left.\int_{Q(z, 2 \sqrt{n} r)^{c}} \mid K(x, y)-K(x, z)\right) a(y) d y d x \\
& \times \int_{Q}\left|b(x)-b_{Q}\right|\left|\int_{Q} \frac{|y-z|^{\delta}}{|x-z|^{n+\frac{\delta}{\alpha}}}\right| a(y)|d y| d x \\
\leqslant & \int_{Q(z, 2 \sqrt{n} r)^{c}} \frac{\left|b(x)-b_{Q}\right|}{|x-z|^{n+\frac{\delta}{\alpha}}} d x .
\end{aligned}
$$

As

$$
\begin{aligned}
& \int_{Q(z, 2 \sqrt{n} r)^{c}} \frac{\left|b(x)-b_{Q}\right|}{|x-z|^{n+\frac{\delta}{\alpha}}} d x \\
& \leqslant \sum_{j=1}^{\infty} \int_{2^{j} r \leqslant|x-z|<2^{j+1} r} \frac{\left|b(x)-b_{Q}\right|}{|x-z|^{n+\frac{\delta}{\alpha}}} d x \\
& \leqslant \sum_{j=1}^{\infty}\left(2^{j+1} r\right)^{-n-\frac{\delta}{\alpha}} \int_{Q\left(z, 2^{j+1} r\right)}\left|b(x)-b_{Q}\right| d x \\
& \leqslant \sum_{j=1}^{\infty} 2^{-(j+1)\left(n+\frac{\delta}{\alpha}\right)} j r^{-n-\frac{\delta}{\alpha}}\left(2^{j+1} r\right)^{n}\|b\|_{\mathrm{BMO}} \\
& \leqslant C\|b\|_{\mathrm{BMO}} r^{-\frac{\delta}{\alpha}} .
\end{aligned}
$$

Thus, we get

$$
\begin{aligned}
\left\|\mu_{2}(\cdot)\right\|_{L^{1}} & \leqslant C\|b\|_{\mathrm{BMO}} r^{\delta\left(1-\frac{1}{\alpha}\right)}\|a\|_{L^{2}}|Q|^{1 / 2} \\
& \leqslant C\|b\|_{\mathrm{BMO}} r^{0}=C\|b\|_{\mathrm{BMO}} .
\end{aligned}
$$


For $\mu_{3}$, as $x \in Q(z, 2 \sqrt{n} r)^{c}$ and $y \in Q(z, r)$, there is $2|y-z|^{\alpha}<2 \sqrt{n} r^{\alpha} \leqslant 2 \sqrt{n} r \leqslant|x-z|$. Thus, using the Hölder inequality and Lemma 1 , we obtain

$$
\begin{aligned}
\left\|\mu_{3}(\cdot, z)\right\|_{L^{1}} \leqslant & \int_{Q(z, 2 \sqrt{n} r)^{c}} \frac{|y-z|^{\delta}}{|x-z|^{n+\frac{\delta}{\alpha}}} \\
& \times\left|b(y)-b_{Q} \| a(y)\right| d y d x \\
\leqslant & r^{\delta} \int_{Q}\left|a(y) \| b(y)-b_{Q}\right| d y \\
& \times \int_{Q(z, 2 \sqrt{n} r)^{c}} \frac{1}{|x-z|^{n+\delta / \alpha}} d x \\
\leqslant & C r^{\delta}\|a\|_{L^{2}}\left(\int_{Q}\left|b(y)-b_{Q}\right|^{2} d y\right)^{1 / 2} \\
& \times \int_{2 \sqrt{n} r}^{\infty} r^{n-1-n-\frac{\delta}{\alpha}} d r \\
\leqslant & C\|b\|_{\mathrm{BMO}} r^{\delta\left(1-\frac{1}{\alpha}\right)}|Q|^{-1 / 2+1 / 2} \\
\leqslant & C\|b\|_{\mathrm{BMO}} .
\end{aligned}
$$

\section{The case $r \leqslant 1$}

For any $H^{1}$ atom $\operatorname{supp}(a) \subset Q=Q(z, r)$ with $0<\epsilon \leqslant$ $r \leqslant 1$, we may decompose $T_{b} a(x)$ as

$$
\begin{aligned}
& T_{b} a(x) \\
& =T_{b} a(x) \chi_{Q\left(z, 2 \sqrt{n} r^{\alpha}\right)}(x)+T_{b} a(x) \chi_{\left(Q\left(z, 2 \sqrt{n} r^{\alpha}\right)\right) c}(x) \\
& =T_{b} a(x) \chi_{Q\left(z, 2 \sqrt{n} r^{a}\right)}(x) \\
& +T\left(a(\cdot)\left(b(\cdot)-b_{Q\left(z, 2 \sqrt{n} r^{\alpha}\right)}\right)\right)(x) \chi_{\left(Q\left(z, 2 \sqrt{n} r^{\alpha}\right)\right)^{c}}(x) \\
& +\chi_{\left(Q\left(z, 2 \sqrt{n} r^{\alpha}\right)\right)^{c}}(x) T\left(\left(b_{Q\left(z, 2 \sqrt{n} r^{\alpha}\right)}-b(\cdot)\right) a\right)(x) \\
& =T_{b} a(x) \chi_{Q\left(z, 2 \sqrt{n} r^{\alpha}\right)}(x) \\
& +T\left(a(\cdot)\left(b(\cdot)-b_{Q\left(z, 2 \sqrt{n} r^{\alpha}\right)}\right)\right)(x) \chi_{\left(Q\left(z, 2 \sqrt{n} r^{\alpha}\right)\right) c}(x) \\
& +\chi_{\left(Q\left(z, 2 \sqrt{n} r^{a}\right)\right) c}(x) \int_{Q}(K(x, y)-K(x, z)) \\
& \times\left(b(y)-b_{Q\left(z, 2 \sqrt{n} r^{\alpha}\right)}\right) a(y) d y \\
& +\chi_{\left(Q\left(z, 2 \sqrt{n} r^{\alpha}\right)\right)^{c}}(x) \\
& \times \int_{Q} K(x, z)\left(b(y)-b_{Q\left(z, 2 \sqrt{n} r^{\alpha}\right)}\right) a(y) d y \\
& :=\mu_{1}(x)+\mu_{2}(x)+\mu_{3}(x, z)+\mu_{4}(x, z) \text {. }
\end{aligned}
$$

Next, we will give the estimates of $\left\|\mu_{i}\right\|_{L^{1}} \quad(i=$ $1,2,3)$ respectively.

$$
\begin{aligned}
& \left\|\mu_{1}(\cdot)\right\|_{L^{1}} \\
& =\int_{Q\left(z, 2 \sqrt{n} r^{\alpha}\right)}|T((b(x)-b(\cdot)) a(\cdot))(x) d x| d x \\
& \leqslant \int_{Q\left(z, 2 \sqrt{n} r^{\alpha}\right)}\left|T a(x) \| b(x)-b_{Q\left(z, 2 \sqrt{n} r^{\alpha}\right)}\right| d x \\
& \quad+\int_{Q\left(z, 2 \sqrt{n} r^{\alpha}\right)}\left|T\left(\left(b(\cdot)-b_{Q\left(z, 2 \sqrt{n} r^{\alpha}\right)}\right) a(\cdot)\right)(x)\right| d x \\
& :=I+I I .
\end{aligned}
$$

For $I$, by the $L^{q} \rightarrow L^{2}$ boundedness of $T$ ((iii) of Definition 1), we have

$$
\begin{aligned}
I= & \int_{Q\left(z, 2 \sqrt{n} r^{\alpha}\right)}\left|T a(x) \| b(x)-b_{Q\left(z, 2 \sqrt{n} r^{\alpha}\right)}\right| d x \\
\leqslant & \left(\int_{Q\left(z, 2 \sqrt{n} r^{\alpha}\right)}\left|b(x)-b_{Q\left(z, 2 \sqrt{n} r^{\alpha}\right)}\right|^{2} d x\right)^{1 / 2} \\
& \times\left(\int_{Q\left(z, 2 \sqrt{n} r^{\alpha}\right)}|T a(x)|^{2} d x\right)^{1 / 2} \\
\leqslant & C\|b\|_{\mathrm{BMO}}\left|Q\left(z, 2 \sqrt{n} r^{\alpha}\right)\right|^{1 / 2}\|a\|_{L^{q}} \\
\leqslant & C\|b\|_{\mathrm{BMO}} r^{n\left(\frac{\alpha}{2}+1 / q-1\right)} .
\end{aligned}
$$

As $\frac{\alpha}{2}+\frac{1}{q}-1=\frac{\alpha}{2}-1+\frac{1}{2}+\frac{\beta}{n} \geqslant \frac{\alpha}{2}-\frac{1}{2}+\frac{1}{2}-\frac{\alpha}{2}=0$ and $0<r \leqslant 1$, we may get

$$
I \leqslant C\|b\|_{\mathrm{BMO}} r^{0}=C\|b\|_{\mathrm{BMO}} .
$$

For $I I$, using the Hölder inequality, Lemma 2 and the $L^{q} \rightarrow L^{2}(1<q<2)$ boundedness of $T$, we have

$$
\begin{aligned}
I I= & \int_{Q\left(z, 2 \sqrt{n} r^{\alpha}\right)}\left|T\left(\left(b(\cdot)-b_{Q\left(z, 2 \sqrt{n} r^{\alpha}\right)}\right) a(\cdot)\right)(x)\right| d x \\
\leqslant & \left(\int_{Q\left(z, 2 \sqrt{n} r^{\alpha}\right)}\left|T\left(\left(b(\cdot)-b_{Q\left(z, 2 \sqrt{n} r^{\alpha}\right)}\right) a(\cdot)\right)(x)\right|^{2} d x\right)^{1 / 2} \\
& \times\left|Q\left(z, 2 \sqrt{n} r^{\alpha}\right)\right|^{1 / 2} \\
\leqslant & C\left\|\left(b(\cdot)-b_{Q\left(z, 2 \sqrt{n} r^{\alpha}\right)}\right) a(\cdot)\right\|_{L^{q}} r^{\frac{\alpha}{2} n} \\
\leqslant & C r^{\frac{\alpha}{2} n}\|a\|_{L^{2}}\left(\int_{Q(z, r)}\left|b(x)-b_{Q\left(z, 2 \sqrt{n} r^{\alpha}\right)}\right|^{\frac{2 q}{2-q}} d x\right)^{\frac{2-q}{2 q}} .
\end{aligned}
$$


Recall $\frac{\alpha}{2}+\frac{1}{q}-1 \geqslant 0$. Then, using the fact $0<\epsilon \leqslant$ $r \leqslant 1$ and Lemma 2, we get

$$
\begin{aligned}
I I \leqslant & r^{\frac{\alpha}{2} n}\|a\|_{L^{2}}\left(\frac{1}{|Q|} \int_{Q} \mid b(y)-b_{\left.\left.Q\left(z, 2 \sqrt{n} r^{\alpha}\right)\right|^{\frac{2 q}{2-q}} d y\right)^{\frac{2-q}{2 q}}}\right. \\
& \times|Q|^{\left(1-\frac{q}{2}\right) \frac{1}{q}} \\
\leqslant & C\|b\|_{\mathrm{BMO}} r^{\frac{\alpha}{2} n} r^{n\left(1-\frac{q}{2}\right) \frac{1}{q}} r^{-\frac{n}{2}} \\
= & C\|b\|_{\mathrm{BMO}} r^{n\left(\frac{\alpha}{2}+1 / q-1\right)} \leqslant C\|b\|_{\mathrm{BMO}} .
\end{aligned}
$$

Combining the above two estimates, we obtain

$$
\left\|\mu_{1}(\cdot)\right\|_{L^{1}} \leqslant C\|b\|_{\mathrm{BMO}} .
$$

For $\mu_{2}$, by the fact $y \in Q(z, r)$ and $x \in Q\left(z, 2 \sqrt{n} r^{\alpha}\right)$, there is $2|y-z|^{\alpha} \leqslant 2 \sqrt{n} r^{\alpha} \leqslant|x-z|$. Thus, we get

$$
\begin{aligned}
\left\|\mu_{2}(\cdot)\right\|_{L^{1}} \leqslant & \int_{Q\left(z, 2 \sqrt{n} r^{\alpha}\right)^{c}}\left|b(x)-b_{Q\left(z, 2 \sqrt{n} r^{\alpha}\right)}\right| \\
& \times \int_{Q(z, r)} \frac{|y-z|^{\delta}|a(y)|}{|x-z|^{n+\frac{\delta}{\alpha}}} d y d x \\
\leqslant & r^{\delta} \int_{Q(z, r)}|a(y)| d y \\
& \times \int_{Q\left(z, 2 \sqrt{n} r^{\alpha}\right)^{c}} \frac{\left|b(x)-b_{Q\left(z, 2 \sqrt{n} r^{\alpha}\right)}\right|}{|x-z|^{n+\frac{\delta}{\alpha}}} d x .
\end{aligned}
$$

As

$$
\begin{aligned}
& \int_{Q\left(z, 2 \sqrt{n} r^{\alpha}\right)^{c}} \frac{\left|b(x)-b_{Q\left(z, 2 \sqrt{n} r^{\alpha}\right)}\right|}{|x-z|^{n+\frac{\delta}{\alpha}}} d x \\
& =\int_{|x-z| \geqslant 2 \sqrt{n} r^{\alpha}} \frac{\left|b(x)-b_{Q\left(z, 2 \sqrt{n} r^{\alpha}\right)}\right|}{|x-z|^{n+\frac{\delta}{\alpha}}} d x \\
& \leqslant \sum_{j=1}^{\infty} \int_{2^{j} \sqrt{n} r^{\alpha} \leqslant|x-z|<2^{j+1} \sqrt{n} r^{\alpha}} \\
& \quad \frac{\left|b(x)-b_{Q\left(z, 2 \sqrt{n} r^{\alpha}\right)}\right|}{|x-z|^{n+\frac{\delta}{\alpha}}} d x \\
& \leqslant \sum_{j=1}^{\infty}\left(2^{j+1} \sqrt{n} r^{\alpha}\right)^{-n-\frac{\delta}{\alpha}} \int_{Q\left(z, 2^{j+1} \sqrt{n} r^{\alpha}\right)} \\
& \leqslant C\|b\|_{\mathrm{BMO}} \sum_{j=1}^{\infty}\left(2^{j+1} \sqrt{n} r^{\alpha}\right)^{-n-\frac{\delta}{\alpha}}(j+1) \\
& \quad \times\left(2^{j+1} \sqrt{n} r^{\alpha}\right)^{n} \\
& =C\|b\|_{\mathrm{BMO}} \sum_{j=1}^{\infty}\left(2^{j+1} \sqrt{n} r^{\alpha}\right)^{-\frac{\delta}{\alpha}}(j+1),
\end{aligned}
$$

we may get

$$
\begin{aligned}
\left\|\mu_{2}(\cdot)\right\|_{L^{1}} \leqslant & C\|b\|_{\mathrm{BMO}} r^{\delta}\|a\|_{L^{2}}|Q|^{1 / 2} \\
& \times \sum_{j=1}^{\infty}\left(2^{j+1} \sqrt{n} r^{\alpha}\right)^{-\frac{\delta}{\alpha}}(j+1) \leqslant C\|b\|_{\mathrm{BMO}} .
\end{aligned}
$$

For $\mu_{3}(x, z)$, note that $2|y-z|^{\alpha} \leqslant 2 \sqrt{n} r^{\alpha} \leqslant$ $|z-x|$, the fact $0<\epsilon \leqslant r \leqslant 1$ with any $\epsilon>0$ and $0<\alpha<1$. Then using (ii) of Definition 1 and Lemma 2, we get

$$
\begin{aligned}
& \left\|\mu_{3}(\cdot, z)\right\|_{L^{1}} \\
& =\int_{Q\left(z, 2 \sqrt{n} r^{\alpha}\right)^{c}} \int_{Q}|K(x, y)-K(x, z)| \\
& \times\left|b(y)-b_{Q\left(z, 2 \sqrt{n} r^{\alpha}\right)}\right||a(y)| d y d x \\
& \leqslant \int_{Q\left(z, 2 \sqrt{n} r^{\alpha}\right)^{c}} \int_{Q} \frac{|y-z|^{\delta}}{|z-x|^{n+\frac{\delta}{\alpha}}} \\
& \times\left|b(y)-b_{Q\left(z, 2 \sqrt{n} r^{\alpha}\right)}\right||a(y)| d y d x \\
& \leqslant r^{\delta} \int_{Q}|a(y)|\left|b(y)-b_{Q\left(z, 2 \sqrt{n} r^{\alpha}\right)}\right| d y \\
& \times \int_{Q\left(z, 2 \sqrt{n} r^{\alpha}\right)^{c}} \frac{1}{|z-x|^{n+\frac{\delta}{\alpha}}} d x \\
& \leqslant C r^{\delta}\|a\|_{L^{2}}|Q|^{1 / 2} \\
& \times\left(\frac{1}{|Q|} \int_{Q}\left|b(y)-b_{Q\left(z, 2 \sqrt{n} r^{\alpha}\right)}\right|^{2} d y\right)^{1 / 2} \\
& \times \int_{2 \sqrt{n} r^{\alpha}}^{\infty} t^{n-1-n-\frac{\delta}{\alpha}} d t \\
& \leqslant C r^{\delta}\|a\|_{L^{2}}|Q|^{1 / 2}\|b\|_{\mathrm{BMO}} \times \int_{2 \sqrt{n} r^{\alpha}}^{\infty} t^{n-1-n-\frac{\delta}{\alpha}} d t \\
& \leqslant C\|b\|_{\text {ВMO }}
\end{aligned}
$$

\section{Proof of Theorem 3}

Finally, we will give the proof of Theorem 3. From Definition 3, we know that for any $f \in H^{1}, f$ can be decomposed by

$$
f=\sum_{j} \lambda_{j} a_{j}
$$

where $a_{j}$ is an $H^{1}$ atom. By using the main results of Ref. 13, we know that the boundedness of $T_{b}$ from $H^{1}$ to $L^{1}$ is equivalent to

$$
\left\|T_{b} a\right\|_{L^{1}} \leqslant C,
$$

where $a$ is an $H^{1}$ atom supported on $Q=Q(z, r)$. 
For case $r>1$. By the definition of $\mu_{4}(x, z)$, the estimates of $\mu_{i}(i=1,2,3)$ and (15), we know that if $T_{b}$ is bounded from $H^{1}$ to $L^{1}$. Thus, we obtain

$$
\left|\int_{Q(z, 2 \sqrt{n} r)^{c}} K(x, z) d x \int_{Q}\left(b_{Q}-b(y)\right) a(y) d y\right| \leqslant C .
$$

By the cancellation conditions of $a$, (16) is equivalent to

$$
\left|\int_{Q(z, 2 \sqrt{n} r)^{c}} K(x, z) d x \int_{Q} b(y) a(y) d y\right| \leqslant C .
$$

On the other hand, if (17) holds, combining (9)(12), we may get (15), which is equivalent to the fact $T_{b} f(x)$ is bounded from $H^{1}$ to $L^{1}$.

Similarly for the case $0<\epsilon \leqslant r \leqslant 1$, we know that the boundedness of $T_{b}$ from $H^{1}$ to $L^{1}$ is also equivalent to

$$
\left|\int_{Q\left(z, 2 \sqrt{n} r^{\alpha}\right)^{c}} K(x, z) d x \int_{Q} b(y) a(y) d y\right| \leqslant C .
$$

Consequently, the proof of Theorem 3 has been finished.

Acknowledgements: We thank the anonymous referees for valuable suggestions and comments which lead to a significant improvement of our manuscript. The research was partially supported by the National Natural Science Foundation of China (Nos. 11561057 and 11501516), the Natural Science Foundation of Jiangxi Province(No. 20151BAB211002) and the Natural Science Foundation of Zhejiang Province under grant(No. LQ15A010003).

\section{REFERENCES}

1. Fefferman C, Stein EM (1972) $H^{p}$ spaces of several variables. Acta Math 129, 137-93.

2. Coifman R (1974) A real variable characterization of $H^{p}$. Studia Math 51, 269-74.

3. Alvarez J, Milman M (1986) $H^{p}$ Continuity Properties of Calderón-Zygmund-type Operators. $J$ Math Anal Appl 118, 63-79.

4. Muckenhoupt B, Wheeden R (1976) Weighted bounded mean oscillation and the Hilbert transform. Studia Math 54, 221-37.

5. Alvarez J, Bagby RJ, Kurtz DS, Pérez C (1993) Weighted estimates for commutators of linear operators. Studia Math 104, 195-209.

6. Lin Y, Lu SZ (2006) Toeplitz operators related to strongly singular Calderón-Zygmund operators. Sci in China, Ser A 49, 1048-64.

7. Coifman R, Rochberg R, Weiss G (1976) Factorization theorems for Hardy spaces in several variables. Ann Math 103, 611-35.
8. Pérez C (1995) Endpoint estimates for commutators of singular integral operators. J Funct Anal 128, 16385.

9. Harboure E, Segovia C, Torrea JL (1997) Boundedness of commutators of fractional and singular integrals for the extreme values of $p$. Illinois $J$ Math 41, 676-700.

10. Lin Y (2007) Storngly singular Calderón-Zygmund Operator and Commutator on Morrey type spaces. Acta Math Sinica (English Ser) 23, 2097-110.

11. Lin Y, Lu SZ (2007) Boundedness of Commutators on Hardy-Type Spaces. Integr Equ Oper Theory 57, 38196.

12. Lin Y, Zhang GM (2016) Weighted estimates for commutators of strongly singular Calderón-Zygmund operators. Acta Math Sinica (English Ser) 32, 1297-311.

13. Yang DC, Zhou Y (2009) A boundedness criterion via atoms for linear operators in Hardy spaces. Constr Approx 29, 207-18. 